Emmer, Martin; Wolling, Jens:

Leben in verschiedenen Welten? : Themenagenden von Offlinern und Onlinern im Vergleich

Original erschienen in:

Kimpeler, Simone; Mangold, Michael; Schweiger, Wolfgang (Hrsg.): Die digitale Herausforderung : zehn Jahre Forschung zur computervermittelten

Kommunikation. - Wiesbaden : VS, Verl. für Sozialwiss. - 2007, S. 239-250.

ISBN (print): 978-3-531-15477-0

ISBN (online): 978-3-531-90649-2

DOI: $\quad$ 10.1007/978-3-531-90649-2_19

URL: $\quad$ http://dx.doi.org/10.1007/978-3-531-90649-2_19

[Gesehen: 18.12.2015] 


\title{
Leben in verschiedenen Welten? Themenagenden von Offlinern und Onlinern im Vergleich
}

\author{
Martin Emmer, Jens Wolling
}

\section{Einleitung}

Eine der wesentlichen Funktionen der Medien für moderne demokratische Gesellschaften ist die Herstellung von Öffentlichkeit für gesellschaftlich relevante Themen und Probleme. Die mediale Behandlung der verschiedenen Issues ist deswegen wichtig, weil sich die Politik vor allem dann eines Themas annimmt, wenn dieses ein Mindestmaß an öffentlicher Aufmerksamkeit erfahren hat. Zum anderen führt die mediale Beachtung dazu, dass die hervorgehobenen Themen zum Gesprächsgegenstand in der Gesellschaft werden. In diesem Zusammenhang ist die massenmediale Berichterstattung insbesondere deswegen von Bedeutung, weil sich die klassischen Massenmedien an einem überschaubaren Set von Relevanzindikatoren (Nachrichtenfaktoren) orientieren, wodurch eine relativ einheitliche Themenagenda entsteht. Diese einheitliche Medienthemenagenda ist eine Voraussetzung dafür, dass sich die Mitglieder der Gesellschaft mit den gleichen Problemen auseinandersetzen und sich darüber untereinander austauschen können. Das daraus entstehende gemeinsame Bewusstsein für die soziale Realität kann zur gesellschaftlichen Integration beitragen.

Im vorliegenden Beitrag soll der Frage nachgegangen werden, ob die Thematisierungsfunktion der Massenmedien durch die wachsende Bedeutung des Internets als politisches Informationsmedium geschwächt wird. Zunächst wird theoretisch erläutert, warum die Nutzung des Internets als Informationsmedium diese Wirkungen haben könnte und welche Forschungsbefunde dazu bereits vorliegen. Anschließend werden dann die Ergebnisse einer empirischen Untersuchung vorgestellt, in der geprüft wurde, ob sich die Themenagenden von Onlinern und Offlinern voneinander unterscheiden.

\section{Theoretischer Hintergrund: Fragmentierung und Agenda Setting}

Bereits seit einigen Jahren wird in der Kommunikationswissenschaft die Frage diskutiert und untersucht, ob das Internet die Fragmentierung der Mediennut- 
zung befördert und auf diese Weise zur Desintegration der Gesellschaft beiträgt (vgl. Holtz-Bacha 1998). Angesichts der enormen Ausweitung und Ausdifferenzierung des Angebots an Informationsmöglichkeiten im Internet, das die Entwicklungen im Bereich des dualen Fernsehsystems und das Wachstum des Zeitschriftenmarktes bei Weitem in den Schatten stellt, erscheint eine solche Entwicklung durchaus plausibel. Es wäre demnach zu erwarten, dass sich die Nutzer, die sich von ihren Voreinstellungen und Kommunikationsbedürfnissen bei der Angebotsauswahl leiten lassen, auf immer mehr Angebote verstreuen. Eine solche Fragmentierung der Mediennutzung des Publikums könnte mittelfristig Auswirkungen auf das zivilgesellschaftliche und politische Engagement haben.

Diese pessimistische These wird insbesondere in den USA seit einiger Zeit intensiv diskutiert (vgl. Putnam 2000). Empirische Ergebnisse, die diese Vermutung stützen, findet man jedoch kaum. Allerdings gibt es auch für die Gegenthese - einer stimulierenden Wirkung des Internets auf das Engagement in Politik und Gesellschaft - relativ wenige Belege (Emmer 2005; Emmer/Vowe 2004). Für eine angemessene Diskussion der These muss zudem unterschieden werden, auf welcher gesellschaftlichen Ebene Desintegrationstendenzen zu vermuten sind: Wird mit negativen Tendenzen hinsichtlich des Zusammenhalts der Familie, der Nachbarschaft oder des Freundeskreises gerechnet (Mikroebene) oder beziehen sich die Befürchtungen auf die Einbindung der Bürger in Vereine und Organisationen (Mesoebene), oder geht es um das Verhältnis der Bürger zu Staat und Gesellschaft (Makroebene). Je nachdem welche Ebene analysiert wird zeigen sich unterschiedliche Effekte. Zudem sollte bei der Diskussion auch konzeptionell präzise zwischen Prozessen gesellschaftlicher Ausdifferenzierung, die zur Pluralität führen, und sozialer Desintegration unterschieden werden. Auch wenn sich die verschiedenen Mitglieder einer Gesellschaft hinsichtlich ihrer Themenpräferenzen, Ansichten und Handlungen deutlich unterscheiden (Pluralität), bedeutet dies keinesfalls, dass sie sich überwiegend von der Gesellschaft abwenden (Desintegration; Wolling 2001).

Neben der Vermutung, dass sich die Ausweitung und Ausdifferenzierung der Kommunikationsangebote im Internet auf die Bereitschaft zum gesellschaftlichen Engagement auswirkt, erscheint insbesondere die These plausibel, dass die zentrale Funktion des Medienjournalismus - die Herstellung und Bereitstellung von Themen für den gesellschaftlichen Diskurs (Rühl 1980: 323) - durch das Internet beeinflusst wird. Die Multioptionalität der Internetkommunikation könnte sich dahingehend auswirken, dass wesentliche Bedingungen, unter denen die Massenkommunikation ihre Wirksamkeit entfaltet, insbesondere Kumulation, Konsonanz und Öffentlichkeitseffekt (Noelle-Neumann 1997), an Bedeutung verlieren. Dies wiederum dürfte Auswirkungen auf die Themen und Ge- 
genstände haben, die Menschen für wichtig halten und denen sie sich bei ihrem gesellschaftlichen Engagement widmen.

Für den gesellschaftlichen Zusammenhalt in einer demokratischen Gesellschaft ist es notwendig, dass eine Verständigung darüber herbeigeführt wird, welche Probleme und Themen in der Öffentlichkeit diskutiert und vom politischen System bearbeitet werden sollen. Bei diesem Verständigungsprozess spielen die Medien eine zentrale Bedeutung. Indem sie aus der Vielfalt der möglichen Themen einige auswählen und diese in der Berichterstattung verstärkt beachten und betonen, fokussieren sie die gesellschaftliche Aufmerksamkeit und stellen sicher, dass ein gesamtgesellschaftlicher Diskurs über diese Aspekte stattfindet (Eichhorn 1996; Erbring et al. 1980; McCombs/Shaw 1972; Rössler 1997).

Aufgrund der Vielzahl unterschiedlicher Informationsangebote im Internet ist dort mit einer größeren Vielfalt in der Themensetzung zu rechnen als bei den herkömmlichen Medienangeboten. Wenn nun eine zunehmende Verlagerung der politischen Mediennutzung auf solche inhaltlich heterogenen Onlineangebote stattfindet, dann könnte die Macht der etablierten Medien, welche die politische Agenda in einer Gesellschaft zu prägen, nach und nach in Frage gestellt werden. In einzelnen Publikumssegmenten könnten sich unterschiedliche Themenprioritäten entwickeln und eine Einigung darüber, was für die Gesellschaft wichtig ist und womit sie sich dementsprechend zu befassen hat, wird schwieriger.

Empirische Ergebnisse zur Nutzung der entsprechenden Onlineangebote scheinen allerdings gegen solche Vermutungen zu sprechen: Trotz der Angebotsvielfalt sind im Netz erhebliche Nutzungskonzentrationen zu beobachten: Die meisten Angebote bleiben weitgehend unbeachtet, während eine kleine Anzahl von Webseiten den Großteil des Traffics auf sich zieht (Loosen 2001). Aber nicht nur diese Nutzungstendenzen sprechen gegen eine Fragmentierung der Agenden. Auch in ersten Wirkungsstudien konnten die Annahmen nicht (vgl. Marr 2002) oder nur begrenzt bestätigen werden (vgl. Schönbach et al. 2005). Allerdings befindet sich die Adaption des Internets immer noch in einem sehr dynamischen Prozess: Die Zahl der Internetnutzer und die Intensität der Internetnutzung nehmen weiterhin zu, auch die Vielfalt der Angebotsformen wächst und unterliegt zudem einem ständigen Wandel. Als besonders relevant erscheint dabei die Tatsache, dass sich im Zuge der Diffusion des Internets neue Gruppen mit spezifischem, auch stark durch das Internet geprägtem Informations- und Kommunikationsverhalten herausbilden (vgl. Emmer et al. 2006), deren Relevanz in aggregierten Datendarstellungen häufig übersehen wird. Die Ergebnisse einer aktuellen Inhaltsanalyse von Quandt (2007) verdeutlichen zudem, dass sich die Themenauswahl bei Print- und Onlineausgabe selbst dann 
beträchtlich unterscheidet, wenn sie vom gleichen Anbieter stammt. Auffällig ist vor allem die breitere Streuung auf verschiedene Themenkategorien bei den Onlinemedien.

Aus diesen Gründen ist anzunehmen, dass bei denjenigen Personen, die sich besonders stark auf politische Informationen aus dem Internet verlassen, andere Themen auf der individuellen Agenda stehen als bei solchen, die sich vorwiegend über herkömmliche (Massen-)Medien informieren (Hypothese 1). $\mathrm{Zu}$ erwarten wäre entsprechend der Fragmentierungshypothese für die Gruppe derjenigen, die vorrangig Internetinformationsangebote nutzt, dass bei ihnen eine größere Vielfalt an Themen festzustellen ist (Hypothese 2). Darüber hinaus ist damit zu rechnen, dass diejenigen, die sich vorwiegend im Netz informieren, aufgrund der globalen Ausrichtung des Internets mehr internationale Themen und weniger lokale und regionale Themen auf ihrer Themenagenda haben (Hypothese 3).

\section{Methode}

Basis der Analyse ist eine bundesweit repräsentative Befragung aus dem Jahr 2005, in der bei 1596 Personen neben den Mediennutzungsgewohnheiten (online und offline) auch weiterführende Aktivitäten politischer Kommunikation (Leserbriefe schreiben, Gespräche über Politik) erhoben wurden. Dabei wurden auch die thematischen Zusammenhänge, die für die befragten Personen bei diesen Kommunikationsaktivitäten jeweils im Mittelpunkt standen, erhoben. Basis der nachfolgenden Analysen ist die Frage, über welches politische Thema sich die Befragten zuletzt im interpersonalen Gespräch unterhalten haben. Im Unterschied zu den meisten Agenda-Setting-Studien wurde also nicht die abstrakte Wichtigkeit eines Themas ermittelt, sondern es wurde die Relevanz unmittelbarer erfasst, indem an die kommunikativen Aktivitäten der Befragten direkt angeknüpft wurde. Um die oben formulierten Hypothesen zu prüfen, wurden die Befragten in Gruppen eingeteilt. Grundlage hierfür war die Häufigkeit der Fernsehnachrichten-Nutzung, die Häufigkeit der Tageszeitungsnutzung sowie die Häufigkeit der Online-Informationssuche. Unterscheidet man die Personen dabei jeweils in Viel- und Wenignutzer, so lassen sie sich in vier Gruppen aufteilen: Personen, die alle Medienangebote häufig nutzen (Allesnutzer), Personen die alle Medienangebote wenig nutzen (Wenignutzer), Personen, 
die vor allem das Internet intensiv nutzen (Onliner) sowie Personen, die nur Fernsehnachrichten und Tageszeitung intensiv nutzen (Offliner). ${ }^{1}$

Tab. 1: Soziodemographisches Profil der Typen (in \%)

\begin{tabular}{lcccc}
\hline & $\begin{array}{c}\text { Wenignutzer } \\
\mathrm{n}=348\end{array}$ & $\begin{array}{c}\text { Offliner } \\
\mathrm{n}=750\end{array}$ & $\begin{array}{c}\text { Allesnutzer } \\
\mathrm{n}=304\end{array}$ & $\begin{array}{c}\text { Onliner } \\
\mathrm{n}=194\end{array}$ \\
\hline Geschlecht & & & & \\
Weiblich & 59,3 & 55,2 & 32,6 & 36,7 \\
Männlich & 40,7 & 44,8 & 67,4 & 63,3 \\
\hline Alter & & & \\
Durchschnittsalter & 41 Jahre & 52 Jahre & 42 Jahre & 35 Jahre \\
$16-25$ & 17,0 & 4,9 & 11,5 & 21,1 \\
$24-45$ & 50,9 & 33,6 & 51,0 & 64,4 \\
$46-65$ & 23,3 & 39,1 & 33,2 & 14,4 \\
+66 & 8,9 & 22,4 & 4,3 & 0,0 \\
\hline Einkommensgruppe & & & & 14,3 \\
Bis $1500 €$ & 23,9 & 10,2 & 2,8 & 46,3 \\
$1500-3000 €$ & 48,3 & 49,0 & 33,9 & 39,4 \\
Über 3000€ & 27,8 & 40,7 & 63,3 & \\
\hline Schulabschluss & & & & 4,1 \\
Hauptschule + niedriger & 23,4 & 25,4 & 9,9 & 67,2 \\
Realschule/POS & 40,9 & 39,0 & 22,8 & \\
Abitur und höher & 33,4 & 35,5 & 64,4 & \\
\hline
\end{tabular}

Hinsichtlich der vorliegenden Forschungsfrage interessiert insbesondere die Gruppe der Onliner: Diese Gruppe ist mit $12 \%$ des Samples die kleinste, sie versammelt mit Abstand die jüngsten Personen mit dem höchsten Bildungsgrad (Tabelle 1). Hinsichtlich Bildung, Geschlechtsverteilung (überwiegend männlich) und auch des Berufstätigkeitsprofils (vorwiegend im Angestelltenverhältnis; in der Tabelle nicht dokumentiert) ähnelt diese Gruppe derjenigen der Allesnutzer, die sich allerdings durch ein höheres Einkommen und ein höheres Alter auszeichnet. Auch die Offliner haben ein prägnantes Profil: Sie versam-

1 Es stand eine Reihe weiterer Variablen zur Verfügung, allerdings führten komplexere Clusteranalyse-Modelle nicht zu wesentlich anderen Gruppierungen der Befragten. Die Operationalisierungen der Variablen sind Online im Internet unter folgender URL einsehbar: www.politische-online-kommunikation.de. 
melt die mit Abstand ältesten Personen (fast ein Drittel Rentner) mit mittlerem Bildungsstand und mittlerem Einkommen und sind stark mit Frauen besetzt. Die Wenignutzer ähneln im Hinblick auf Geschlecht und Bildung den Offlinern, sind aber deutlich jünger und verfügen über ein geringeres Einkommen. In dieser Gruppe findet sich auch der höchste Anteil von Arbeitern und Arbeitslosen.

Im folgenden Abschnitt werden einige dieser Variablen als Faktoren, die möglicherweise die Wirkung der spezifischen Mediennutzung auf die Beurteilung der Themenwichtigkeit modifizieren, herangezogen.

\section{Ergebnisse}

Um die erste der oben formulierten Hypothesen (Onliner haben andere Themen auf der Agenda) zu prüfen, müssen zunächst die von den Befragten genannten Themen in Kategorien zusammengefasst werden. Da von jeder befragten Person nur eine einzige Themennennung vorliegt, ist es nicht möglich, daraus individuelle Themenranglisten $\mathrm{zu}$ erstellen und $\mathrm{zu}$ vergleichen. Solche Ranglisten sind nur auf Aggregatebene der vier Gruppen möglich (Tabelle 2).

Tab. 2: Rangliste der wichtigsten Themen

\begin{tabular}{lcccc}
\hline Rang & Wenignutzer & Offliner & Allesnutzer & Onliner \\
\hline 1 & $\begin{array}{c}\text { Arbeitmarktre- } \\
\text { form/Hartz IV }\end{array}$ & $\begin{array}{c}\text { Arbeitsmarktre- } \\
\text { form/Hartz IV }\end{array}$ & $\begin{array}{c}\text { Arbeitsmarktre- } \\
\text { form/Hartz IV }\end{array}$ & $\begin{array}{c}\text { Arbeitsmarktre- } \\
\text { form/Hartz IV }\end{array}$ \\
\hline \multirow{2}{*}{3} & $\begin{array}{c}\text { Arbeitsmarkt- } \\
\text { situation }\end{array}$ & $\begin{array}{c}\text { aktuelle deutsche } \\
\text { Politik }\end{array}$ & $\begin{array}{c}\text { Arbeitsmarkt- } \\
\text { situation }\end{array}$ & $\begin{array}{c}\text { Arbeitsmarkt- } \\
\text { situation }\end{array}$ \\
\hline \multirow{2}{*}{$\begin{array}{c}\text { Gesundheits- } \\
\text { politik }\end{array}$} & Bildungspolitik & $\begin{array}{c}\text { Gesundheits- } \\
\text { politik }\end{array}$ & $\begin{array}{c}\text { Normen, Werte, } \\
\text { Zeitgeschehen }\end{array}$ \\
\hline 5 & $\begin{array}{c}\text { Normen, Werte, } \\
\text { Zeitgeschehen }\end{array}$ & $\begin{array}{c}\text { Tsunami- } \\
\text { Katastrophe }\end{array}$ & $\begin{array}{c}\text { Regional-/ } \\
\text { Kommunalpolitik }\end{array}$ & $\begin{array}{c}\text { Tsunami- } \\
\text { Katastrophe }\end{array}$ \\
\hline & Weltgeschehen & Kommunalpolitik & $\begin{array}{c}\text { Sozialpolitik } \\
\text { (Rente, Familie) }\end{array}$ & Finanzpolitik \\
\hline
\end{tabular}

Es zeigt sich, dass zum Befragungszeitpunkt im Januar 2005 die Hartz-IVDebatte und die Arbeitsmarktsituation in allen vier Gruppen das mit Abstand dominierende Thema war (im Durchschnitt aller Gruppen nannte jeder dritte Befragte ein Gesprächsthema aus diesem Komplex). Auf den weiter hinten liegenden Rängen des politischen Themenspektrums sind dann jedoch einige Unterschiede festzustellen. Allerdings ist es hier sinnvoller, die Prozentwerte statt der Rangplätze zu vergleichen, da sich manche Ränge innerhalb der Gruppen nur noch im Nachkommabereich voneinander unterscheiden. 
Auch im Hinblick auf die dritte Hypothese (Onliner haben mehr internationale Themen) zeigen sich beim Vergleich der Rangreihen zunächst nur schwache Belege: Zwar spielt Regionalpolitik tatsächlich lediglich in den beiden Gruppen, die in stärkerem Maße herkömmliche Medien nutzen, eine nennenswerte Rolle - anderseits werden aber internationale Themen wie die TsunamiKatastrophe oder allgemein weltpolitische Themen nicht nur von den Onlinern, sondern auch von den Offlinern recht häufig genannt.

Da die Auswertung anhand der Rangreihen schnell an Grenzen stößt, werden im nächsten Schritt die Prozentanteile der Themennennungen bei den vier Mediennutzer-Typen verglichen. Dabei muss berücksichtigt werden, dass sich die vier Nutzergruppen auch hinsichtlich ihrer soziodemographischen Struktur unterschieden. Wie oben verdeutlicht, differieren die Gruppen vor allem bezüglich des Alters und der Bildung. Es ist durchaus denkbar, dass sich diese unterschiedlichen Strukturmerkmale der Gruppen auch auf die Relevanzeinschätzungen der Themen auswirken. Deswegen werden die Auswertungen jeweils getrennt für die Befragten mit hohem und niedrigem Bildungsgrad sowie für jüngere und ältere Personen durchgeführt. Auf diese Weise kann sichergestellt werden, dass Unterschiede zwischen den Gruppen nicht auf diese Drittvariablen zurückzuführen sind.

Ein erster Analyseschritt (Tabelle 3) ergibt, dass etwa ein Drittel der Wenignutzer kein Gesprächsthema genannt hat, bei den Allesnutzern ist es nur ein Fünftel. Offliner und Onliner liegen dazwischen. Sowohl Alter als auch Bildung scheinen hier einen gewissen Einfluss auszuüben: Unter den Älteren und höher Gebildeten gibt es mehr Personen, die ein politisches Gesprächsthema angeben konnten, über das sie sich unterhalten haben. Für unsere Argumentation entscheidend ist aber, ob sich das grundlegende Muster der Verteilung in den vier Gruppen bedeutsam ändert. Dies ist nicht der Fall. Die Allesnutzer liegen immer an erster und die Onliner immer an zweiter Stelle. Wenignutzer und Offliner liegen in allen vier Gruppen eng beieinander. 
Tab. 3: Präferenzen für Themenbereiche nach Bildung \& Alter (in \%)

\begin{tabular}{|c|c|c|c|c|}
\hline & $\begin{array}{l}\text { Wenignutzer } \\
n=348\end{array}$ & $\begin{array}{l}\text { Offliner } \\
\mathrm{n}=750\end{array}$ & $\begin{array}{l}\text { Allesnutzer } \\
n=304\end{array}$ & $\begin{array}{l}\text { Onliner } \\
\mathrm{n}=194\end{array}$ \\
\hline Mind. 1 Thema genannt & 68,2 & 71,0 & 80,7 & 75,5 \\
\hline Bildung niedrig & 64,4 & 68,3 & 77,8 & 73,4 \\
\hline Bildung hoch (Abitur) & 76,1 & 76,2 & 82,1 & 76,3 \\
\hline Alter niedrig & 62,7 & 60,6 & 73,6 & 72,4 \\
\hline Alter hoch $(>35)$ & 72,0 & 72,6 & 83,6 & 80,9 \\
\hline Internationale Themen & 15,4 & 11,2 & 14,6 & 23,0 \\
\hline Bildung niedrig & 16,0 & 10,3 & 11,9 & 17,0 \\
\hline Bildung hoch (Abitur) & 14,6 & 12,9 & 16,3 & 25,0 \\
\hline Alter niedrig & 23,8 & 12,3 & 19,4 & 28,9 \\
\hline Alter hoch $(>35)$ & 9,7 & 11,1 & 12,9 & 16,7 \\
\hline Regional-/Kommunalpolitik & 2,1 & 6,9 & 6,9 & 2,0 \\
\hline Bildung niedrig & 1,3 & 7,3 & 6,0 & 4,3 \\
\hline Bildung hoch (Abitur) & 3,4 & 5,9 & 7,5 & 1,0 \\
\hline Alter niedrig & 2,4 & 5,3 & 6,0 & 2,6 \\
\hline Alter hoch $(>35)$ & 1,9 & 7,1 & 7,3 & 1,4 \\
\hline Sozial-/Gesundheitspolitik & 12,9 & 13,9 & 8,6 & 4,0 \\
\hline Bildung niedrig & 14,0 & 12,2 & 9,5 & 2,1 \\
\hline Bildung hoch (Abitur) & 11,3 & 16,8 & 8,2 & 5,0 \\
\hline Alter niedrig & 7,2 & 7,0 & 1,5 & 2,6 \\
\hline Alter hoch $(>35)$ & 16,2 & 14,7 & 11,3 & 5,6 \\
\hline Politisches System & 7,1 & 8,2 & 10,6 & 2,8 \\
\hline Bildung niedrig & 7,3 & 9,7 & 7,2 & 0,0 \\
\hline Bildung hoch (Abitur) & 6,7 & 6,0 & 12,5 & 4,0 \\
\hline Alter niedrig & 6,0 & 8,8 & 10,5 & 1,3 \\
\hline Alter hoch $(>35)$ & 7,8 & 7,9 & 10,6 & 4,2 \\
\hline
\end{tabular}

Betrachtet man die von den Befragten genannten politischen Gegenstände genauer, so zeigt sich bei einer ersten groben Aufteilung in nationale und internationale Themen, dass in der Gruppe der Onliner internationale Themen im Verhältnis zu nationalen Themen eine wesentlich größere Rolle spielen als in den anderen drei Gruppen. Dieser Trend, den man als globalisierende Wirkung des Internets deuten kann, wird allerdings durch den Einfluss von Bildung und Alter relativiert. Bei den Älteren und den Personen mit niedrigerer Bildung sind die 
Unterschiede zwischen den Nutzergruppen deutlich schwächer, verschwinden aber auch dort keinesfalls vollständig.

Wenn man noch einen Schritt weitergeht, um eine etwas feinere Unterteilung der Themen vorzunehmen, wird die Analyse etwas schwieriger, weil die Fallzahlen aufgrund des Erhebungsverfahrens (von jeder Person liegt nur eine Themennennung vor) schnell sehr klein werden. Einige Themenkategorien, die für die Prüfung der oben aufgestellten Hypothesen von Relevanz sind, lassen sich jedoch noch weiter ausdifferenzieren und analysieren. So bestätigt sich bei der Nennung lokal- und regionalpolitischer Themen der Trend einer eher globalisierenden Wirkung des Internets zumindest ansatzweise: In den Gruppen, die sich (auch) stärker auf klassische Medien stützen (Offliner und Allesnutzer), werden regionale Themen häufiger genannt.

Einer der Themenkomplexe, der in den Medien im Befragungszeitraum eine relativ große Rolle gespielt hat, ist der Bereich der Sozial- und Gesundheitspolitik. Auch dieser Themenbereich, der eher von nationaler Bedeutung ist, wird von den Nutzern der klassischen Medien deutlich häufiger thematisiert. Erwartungsgemäß hat vor allem das Alter der Befragten einen Effekt auf die Auswahl dieses Themenkomplexes als Gesprächsstoff. Aber auch nach Kontrolle von Alter und Bildung erweisen sich vor allem die Onliner als diejenigen, in deren Gesprächen dieses Thema eine nur sehr untergeordnete Rolle spielt.

Zuletzt soll noch ein Themenbereich betrachtet werden, der im Kontext politischer Mediennutzung und der Politikverdrossenheitsdebatte häufig untersucht wird, nämlich die Thematisierung des politischen Systems und dessen Leistungsfähigkeit generell, inklusive politischer Skandale (in den Befragungszeitraum fielen u.a. die Rücktritte von Laurenz Mayer, Monika Hohlmeier und Cornelia Pieper im Zusammenhang mit verschiedenen Affären). Hier führen wiederum die Gruppen der Allesnutzer und der Offliner. Es ist auffällig, wie wenig Nennungen hier aus der Gruppe der Onliner kommen. Alter und Bildung haben auch hier wieder einen gewissen modifizierenden Einfluss, aber auch in diesem Fall verändert sich das beschriebene Ergebnismuster nur partiell.

Um schließlich die Frage nach der Vielfalt der Themen (Hypothese 2) in den vier untersuchten Gruppen zu beantworten, wurden zwei Kennwerte berechnet: der 'Diversity-Index' und das 'Standardisierte Entropie-Maß'.2 Ein Blick auf die Ergebnisse (Tabelle 4) zeigt, dass sich die Nutzergruppen kaum unterscheiden.

Beide Maßzahlen können Werte zwischen 0 und 1 annehmen, wobei 1 bedeutet, dass eine maximale Vielfalt zu verzeichnen ist, während 0 maximale Homogenität anzeigt. Maximale Vielfalt wäre gegeben, wenn alle Themenkategorien von gleich vielen Personen genannt worden wären, bei maximaler Homogenität wäre erreicht, wenn alle Befragten das gleiche Thema genannt hätten (Franzmann/Wagner 1999: 78ff.). 
Tab. 4: Vielfaltsindikatoren

\begin{tabular}{lcccc}
\hline & Wenignutzer & Offliner & Allesnutzer & Onliner \\
\hline Diversity-Index & 0,90 & 0,90 & 0,92 & 0,89 \\
Entropie-Maß & $\mathbf{0 , 6 4}$ & 0,64 & 0,67 & $\mathbf{0 , 6 2}$ \\
\hline
\end{tabular}

Zudem findet man keinerlei Hinweis darauf, dass sich die Gruppe der Onliner durch eine besonders große Heterogenität auszeichnet. Ganz im Gegenteil, beide Kennwerte sind in dieser Gruppe am niedrigsten. Am heterogensten ist vielmehr die Gruppe der Allesnutzer.

\section{Resümee}

Ziel dieser Analyse war es, Hypothesen über mögliche unterschiedliche politische Themenpräferenzen von Menschen unterschiedlicher Mediennutzungsmuster zu prüfen. Im Mittelpunkt stand dabei die Annahme, dass ein intensiver Rückgriff auf Online-Quellen bei gleichzeitiger Abwendung von herkömmlichen Informationsquellen zu deutlich anderen, insbesondere vielfältigeren und stärker globalen Themenpräferenzen führen könnte.

Die Analyse der vorliegenden Befragungsdaten aus dem Jahr 2005 zeigt, dass diese Annahmen nicht in dem Maße bestätigt wurden, wie es die vorliegende Literatur z.T. nahe legt. So zeigen sich insgesamt eher geringe Unterschiede bei Themeninteressen von On- und Offlinern. Auch bei den Vielfaltsindikatoren sind die Unterschiede eher schwach ausgeprägt. Hinsichtlich der Regionalität/Internationalität politischer Themen zeigen sich zwar einige Unterschiede, die allerdings in ihrer Ausprägung ebenfalls eher schwach und zumindest teilweise von den intervenierenden Variablen Bildung und Alter beeinflusst sind.

Diese Befunde stehen allerdings unter verschiedenen Vorbehalten: So ist die gewählte Methode mit erst nachträglicher Kategorienbildung auf Basis offener Antworten der Befragten relativ stark abhängig von der aktuellen politischen Situation und schränkt die Auswertungsmöglichkeiten ein. Zudem stellt sie nur eine Momentaufnahme aus dem Januar 2005 dar. Erst eine vergleichende Analyse über mehrere Zeitpunkte hinweg könnte zeigen, wie stabil bzw. wie stark situationsabhängig die ermittelten Themenpräferenzen sind. Dies gilt ebenso für die der Analyse zugrunde gelegten Mediennutzungsmuster, die sich insbesondere im Bereich der Online-Angebote ständig verändern. Hinzu kommt, dass ein Nachweis von Medienwirkungen durch die Analyse von Querschnittsdaten ohne Einbeziehung von Inhaltsanalysedaten keinesfalls möglich ist. 
Es lässt sich vorläufig festhalten, dass eine verstärkte Nutzung des Internets für die politische Kommunikation offenbar teilweise veränderte Themenprofile der Mediennutzer zur Folge hat, insgesamt jedoch die Realitätswahrnehmung nicht dramatisch beeinflusst: Die deutschen Online- und Offline-Mediennutzer leben (noch?) nicht in verschiedenen Welten. Da jedoch in der Gruppe der Onliner die jüngeren Kohorten sehr stark vertreten sind, deutet dies auf eine allmähliche aber grundsätzliche Veränderung klassischer Mediennutzungsmuster bei der mit dem Internet aufgewachsenen Generation hin. Mittel- und langfristig könnte dies durchaus dazu führen, dass sich auch in breiteren Bevölkerungsschichten die Themenpräferenzen verschieben.

\section{Literatur}

Eichhorn, Wolfgang (1996): Agenda-Setting-Prozesse: eine theoretische Analyse individueller und gesellschaftlicher Themenstrukturierung. München: Reinhard Fischer.

Emmer, Martin (2005): Politische Mobilisierung durch das Internet? Eine kommunikationswissenschaftliche Untersuchung zur Wirkung eines neuen Mediums. München: Reinhard Fischer.

Emmer, Martin/Füting, Angelika/Vowe, Gerhard (2006): Wer kommuniziert wie über politische Themen? Eine empirisch basierte Typologie individueller politischer Kommunikation. In: Medien \& Kommunikationswissenschaft 54.2. 216-236.

Emmer, Martin/Vowe, Gerhard (2004): Mobilisierung durch das Internet? Ergebnisse einer empirischen Längsschnittuntersuchung zum Einfluss des Internets auf die politische Kommunikation der Bürger. In: Politische Vierteljahresschrift 45.2. 191-211.

Erbring, Lutz/Goldenberg, Edie N./Miller, Arthur H. (1980): Front Page News and Real World Cues: A New Look at Agenda Setting by the Media. In: American Journal of Political Science 24.1. 16-49.

Franzmann, Gabriele/Wagner, Michael (1999): Heterogenitätsindizes zur Messung der Pluralität von Lebensformen und ihre Berechnung in SPSS. In: ZA-Information 44. 75-95.

Gellner, Winand/von Korff, Fritz (Hrsg.) (1998): Demokratie im Internet. Baden-Baden: Nomos.

Holtz-Bacha, Christina (1998): Fragmentierung der Gesellschaft durch das Internet? In: Gellner/von Korff (Hrsg.) (1998): 219-226.

Loosen, Wiebke (2001): Das Ende der Verheißungen? Technisch und ökonomisch determinierte Beschränkungen des "freien" Informationszugangs im World Wide Web. In: MaierRabler/Latzer (Hrsg.) (2001): 71-93.

Maier-Rabler, Ursula/Latzer, Michael (Hrsg.) (2001): Kommunikationskulturen zwischen Kontinuität und Wandel. Konstanz: UVK.

Marr, Mirko (2002): Politikbildung durch Internetnutzung: Ein Vergleich von Off- und Onlinern. Solothurn. 
McCombs, Maxwell E./Shaw, Donald L. (1972): The Agenda-Setting Function of Mass Media. In: Public Opinion Quarterly 36.2. 176-187.

Noelle-Neumann, Elisabeth (1997): Massenmedien und sozialer Wandel - Methodenkombination in der Wirkungsforschung. In: Zeitschrift für Soziologie 8.2. 164-182.

Putnam, Robert D. (2000): Bowling Alone: The Collapse and Revival of American Community. New York: Simon \& Schuster.

Quandt, Thorsten (2007): (K)Ein völlig neuer Journalismus? Eine Inhaltsanalyse tagesaktueller Nachrichtenangebote im WWW. München. Aufsatz in Vorbereitung.

Rössler, Patrick (1997): Agenda-Setting: theoretische Annahmen und empirische Evidenzen einer Medienwirkungshypothese. Opladen: Westdeutscher Verlag.

Rühl, Manfred (1980): Journalismus und Gesellschaft. Bestandsaufnahme und Theorienetwurf. Mainz: v. Hase \& Köhler.

Schönbach, Klaus/de Waal, Ester/Lauf, Edmund (2005): Online and Print Newspapers: Their Impact on the Extent of the Perceived Public Agenda. In: European Journal of Communication 20.2. $245-258$.

Wolling, Jens (2001): Auswirkungen des Internets auf Integration und Pluralität. Konzeptionelle Überlegungen und empirische Befunde. In: Maier-Rabler/Latzer (Hrsg.) (2001): 127-143. 